AIAA-94-1195-CP

\title{
Free-floating Dual-arm Robots for Space Assembly
}

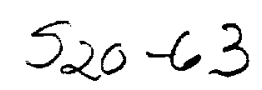

\author{
Sunil Kumar Agrawal, Asst. Professor \\ M.Y. Chen, Graduate Student \\ Department of Mechanical Engineering \\ Ohio University, Athens, OH 45701.
}

\begin{abstract}
Freely moving systems in space conserve linear and angular momentum. As moving systems collide, the velocities get altered due to transfer of momentum. The development of strategies for assembly in a free-floating work environment requires a good understanding of primitives such as self motion of the robot, propulsion of the robot due to onboard thrusters, docking of the robot, retrieval of an object from a collection of objects, and release of an object in an object pool. The analytics of such assemblies involve not only kinematics and rigid body dynamics but also collision and impact dynamics of multibody systems. In an effort to understand such. assemblies in zero gravity space environment, we are currently developing at Ohio University a free-floating. assembly facility with a dual-arm planar robot equipped with thrusters, a free-floating material table, and a freefloating assembly table. The objective is to pick up workpieces from the material table and combine into prespecified assemblies. This paper presents analytical models of assembly primitives and strategies for overall assembly. A computer simulation of an assembly is developed using the analytical models. The experiment facility will be used to verify the theoretical predictions.
\end{abstract}

\section{Introduction}

Over the last two decades, a number of studies have been reported on motion planning of free-floating robots ([10], [7], [12], [13], [9], [11], [1], [2], [3], [4]). However, none of these studies dealt with analytics of entire assemblies in a free-floating work environment using free-floating robots. The analytics of these assemblies involve not only kinematics and rigid body dynamics but also collision and impact dynamics of multibody systems. In an effort to understand assemblies in zero gravity space environment, we are currently developing at Ohio University a free-floating assembly facility with a dual-arm planar robot equipped with thrusters, a free-floating material table, and a free-floating assembly table.
The objective of this experiment testbed is to verify the analytics of assemblies in free-floating work environment. This paper is organized in the following way: An outline of the free-floating robot facility of Ohio University, its analytical descriptions, and kinematics are presented in Section 2. The analytical models of the assembly primitives such as self motion, propulsion, docking, pickup, and release are described in Section 3. An assembly problem is discussed in Section 4. An outline of a general purpose simulation program FLOAT is described in Section 5 which is designed to study strategies of assembly.

\section{Free-Floating Facility}

\subsection{Physical Setup}

The free-floating robot facility of Ohio University consists of a free-floating dual-arm planar robot, a free-floating material table, and a free-floating assembly table. A photograph of the dual-arm freefloating robot is shown in Figure 1. Each of these three units rests on a granite surface supported by four air bearings. Regulated supply of Nitrogen from pressurized cylinders float the units on the granite surface. The robot consists of two arms, each with 3 revolute joints and a prismatic joint. The 3 revolute joints provide the end-effector full mobility in the plane. The prismatic joints are used to move the arms normal to the table. The 8 joints are driven by dc servomotors fitted with optical encoders. A PC 386 motherboard with power from rechargable lead-acid batteries sits on the base of the robot. The motherboard is connected to an 8axis motion control board and a DAS board. Two quad-thrusters are mounted on the base which are controlled by solenoid valves that use air supply from the tank [5]. The robot communicates with a host PC 486 workstation through a radio-wave modem. Two light bulbs fixed on the base of the robot are tracked by an overhead optoelectronic sensor 


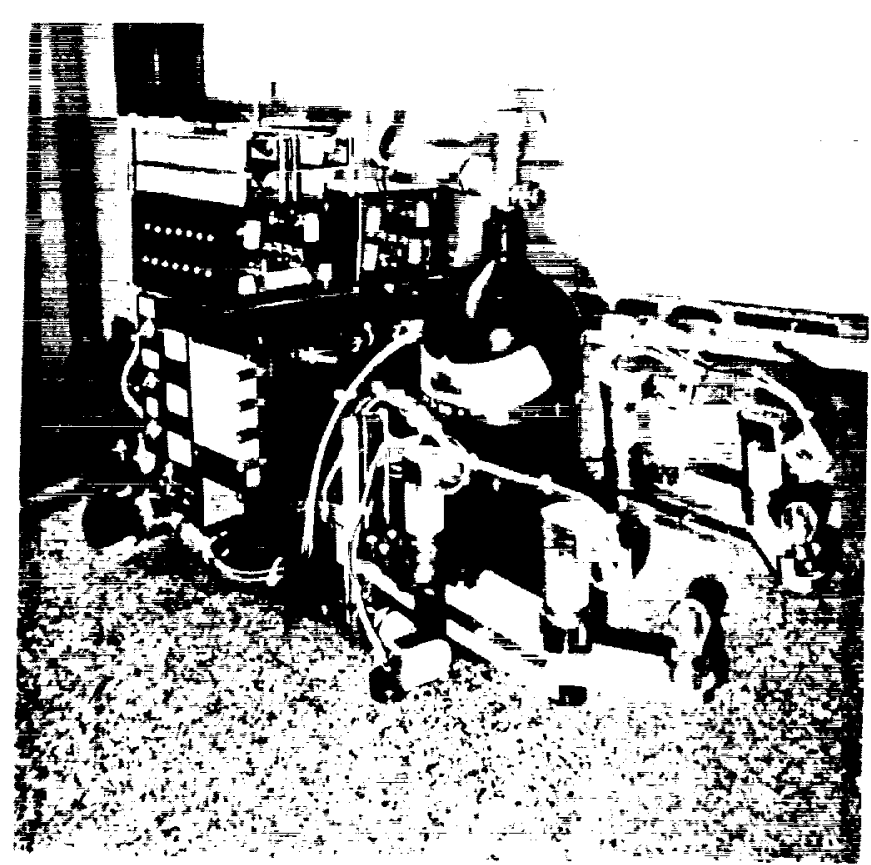

Figure 1: A photograph of a free-floating dual-arm planar robot built at Ohio University.

consisting of a Position Sensitive Detector (PSD) fixed at the focal plane of a TV lens [6]. The PSD sensor is connected to a host PC 486 computer and the voltage outputs of the sensor are calibrated to the position of light bulbs on the table. The sensor provides a feedback of base position and orientation.

The material table also has a pressurized Nitrogen tank which provides the gas needed to float the table on the granite surface. The material table has two light bulbs which are used to feedback the position and orientation of the table to the host PC 486 computer. This table has polished grooves to place the work pieces for assembly. The assembly table has a setup for floatation and position feedback similar to the material table. The grooves in the assembly table are designed to store subassemblies and final assemblies.

One of the assumptions made in this paper is that the joints of the robot are locked during propulsion, docking, pickup, and release and are unlocked during self motion.

\subsection{Analytical Modeling}

From an analytical standpoint, the free-floating facility consists of the following three systems: (i) the robot system (RS), (ii) the material table system (MS), and (iii) the assembly table system (AS). These three systems are made up from the following units: (i) the dual-arm robot, (ii) the material table, (iii) the assembly table, and (iv) the individual work pieces $\left(W_{i}\right)$. The definitions of these three

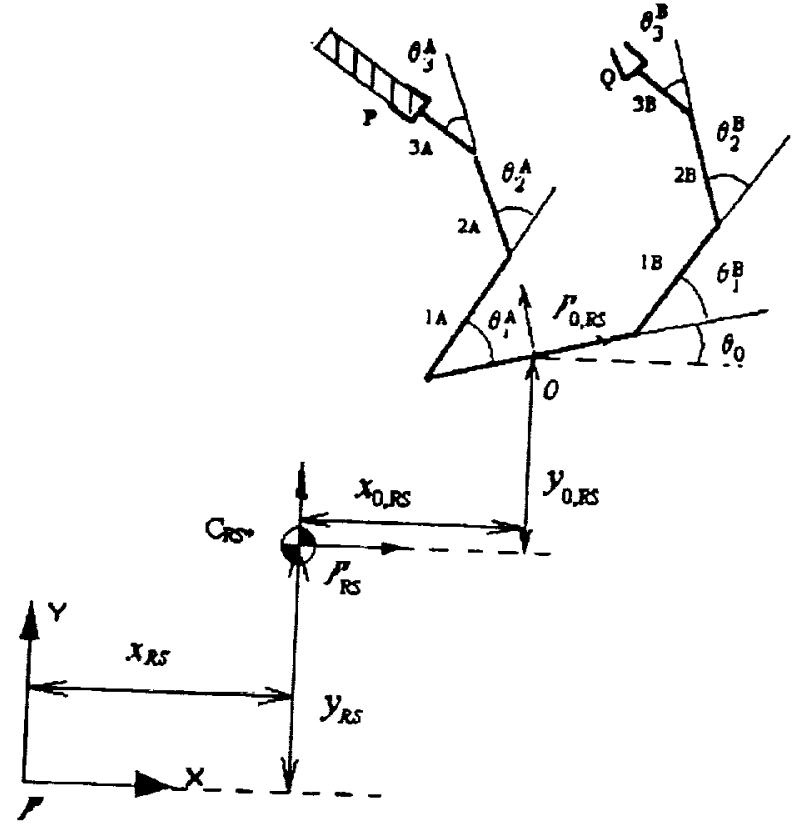

Figure 2: An analytical model of a dual-arm free-floating planar robot.

systems change as the assembly progresses and the workpieces are passed from one system to the other.

\subsubsection{Robot System}

The robot system (RS) consists of the robot and workpieces held by the end-effectors. The robot consists of seven links and its two arms are labeled as $A$ and $B$. The base is labeled as 0 , the three links of arm $A$ are $1 A, 2 A$, and $3 A$, and the three links of arm $B$ are $1 B, 2 B$, and $3 B$. The gripper points on the end-effectors of $A$ and $B$ are respectively $P$ and $Q$. These grippers are designed to catch the workpieces so that they extend outwards from the end-effector links. The joint angles of arm $A$ are $\theta_{1}^{A}$, $\theta_{2}^{A}, \theta_{3}^{A}$ and of $\mathrm{B}$ are $\theta_{1}^{B}, \theta_{2}^{B}$, and $\theta_{3}^{B}$. The prismatic joints in the two arms are not modeled because they are used only periodically to lower and lift the endeffectors. A coordinate frame $\mathcal{F}$ is fixed inertially to the granite table parallel to the edges. A coordinate frame $\mathcal{F}_{R S}$ is fixed at the center of mass of the robot system $C_{R S *}$ with axes parallel to the axes of $\mathcal{F} . \mathcal{F}_{0, R S}$ is fixed on the base link at the midpoint of the two joints located on it. The origin of $\mathcal{F}_{R S}$ is described relative to $\mathcal{F}$ by the coordinate variables $x_{R S}$ and $y_{R S}$. The coordinates $x_{0, R S}$ and $y_{0, R S}$ describe the origin of $\mathcal{F}_{0}$ relative to $\mathcal{F}_{R S} . \theta_{0}$ is the relative orientation between the $\mathrm{X}$ axes of $\mathcal{F}_{0, R S}$ and $\mathcal{F}$. Each link has a mass $m_{i}^{j}$, a center of mass $C_{i *}^{j}$, and a moment of inertia $I_{i}^{j}$ for $i=1,2,3$ and $j=A, B$. These quantities for the base link are respectively $m_{0}, C_{0 *}$, and $I_{0}$. The robot system is shown in Figure 2. During assembly, the inertial 


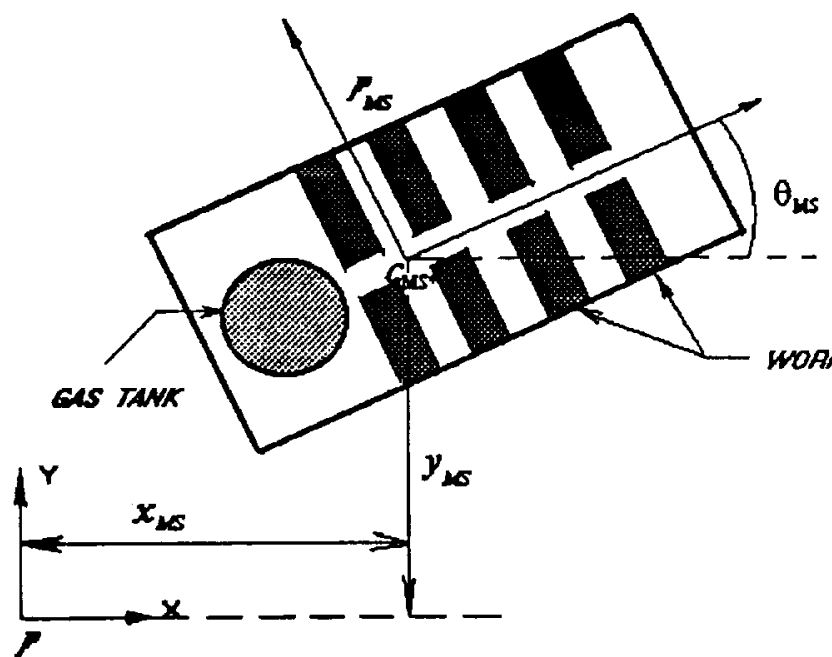

Figure 3: A sketch of the free-floating material table.

parameters of the links $3 \mathrm{~A}$ and $3 \mathrm{~B}$ are computed from the current definition of the robot system.

In summary, the robot system is described by 11 variables: $x_{R S}, y_{R S}, x_{0, R S}, y_{0, R S}, \theta_{0}, \theta_{1}^{A}, \theta_{2}^{A}, \theta_{3}^{A}$, $\theta_{1}^{B}, \theta_{2}^{B}$, and $\theta_{3}^{B}$. During self motion, the 6 joint angles of the robot are actively controlled and during propulsion, docking, and pickup, these 6 joints are locked.

\subsubsection{Material Table System}

The material table system (MS) has 8 slots for the workpieces $W_{1}, \ldots, W_{8}$ to rest. The center of mass of the current system is labeled as $C_{M S *}$. A coordinate frame $\mathcal{F}_{M S}$ is fixed to MS at $C_{M S *}$ parallel to the edges of the material table. The origin of $\mathcal{F}_{M S}$ is described relative to $\mathcal{F}$ by the variables $x_{M S}$ and $y_{M S} . \theta_{M S}$ is the relative angle between the $\mathrm{X}$ axes of the frames $\mathcal{F}$ and $\mathcal{F}_{M S}$. A sketch of the material table system is shown in Figure 3.

\subsubsection{Assembly Table System}

The assembly table has slots to store the intermediate and final assemblies. The center of mass of AS is at $C_{A S *}$. A coordinate frame $\mathcal{F}_{A S}$ is fixed at the center of mass $C_{A S *}$ with axes parallel to the edges of the assembly table. $\mathcal{F}_{A S}$ is described relative to $\mathcal{F}$ by 3 coordinate variables $x_{A S}, y_{A S}$, and $\theta_{A S}$. A sketch of the assembly table system is shown in Figure 4.

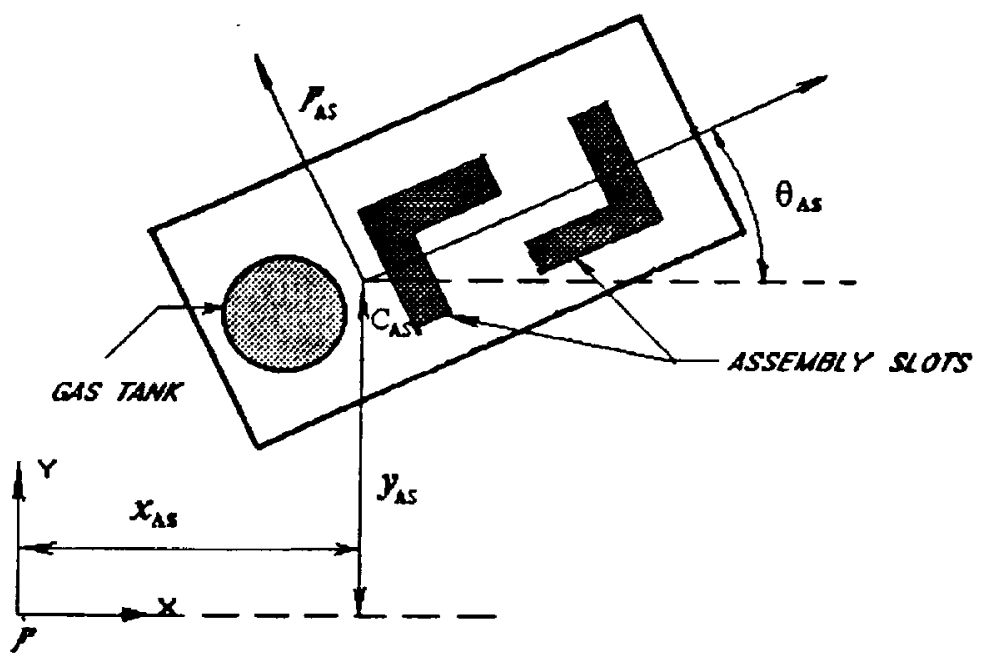

Figure 4: A sketch of the free-floating assembly table.

\subsection{Kinematics}

\subsubsection{Robot System during Free Motion}

With the assumption that the center of mass of RS is at $C_{R S_{*}}$, the 11 variables must satisfy 2 constraints:

$$
\begin{aligned}
& m_{0} \mathbf{r}_{0 *}+m_{1}^{A} \mathbf{r}_{1 *}^{A}+m_{2}^{A} \mathbf{r}_{2 *}^{A}+m_{3}^{A} \mathbf{r}_{3 *}^{A}+m_{1}^{B} \mathbf{r}_{1 *}^{B}+m_{2}^{B} \mathbf{r}_{2 *}^{B} \\
& +m_{3}^{B} \mathbf{r}_{3 *}^{B}=m_{R S} \mathbf{r}_{R S *}
\end{aligned}
$$

where the position vectors are to the center of mass of the respective links in $\mathcal{F}$. On time differentiating the above equation and collecting the terms, it can be written in the following form:

$$
\begin{aligned}
& a_{11} \dot{x}_{0, R S}+a_{13} \dot{\theta}_{0}+a_{14} \dot{\theta}_{1}^{A}+a_{15} \dot{\theta}_{2}^{A}+a_{16} \dot{\theta}_{3}^{A}+a_{17} \dot{\theta}_{1}^{B} \\
& \quad+a_{18} \dot{\theta}_{2}^{B}+a_{19} \dot{\theta}_{3}^{B}=0 \\
& a_{22} \dot{y}_{0, R S}+a_{23} \dot{\theta}_{0}+a_{24} \dot{\theta}_{1}^{A}+a_{25} \dot{\theta}_{2}^{A}+a_{26} \dot{\theta}_{3}^{A}+a_{27} \dot{\theta}_{1}^{B} \\
& +a_{28} \dot{\theta}_{2}^{B}+a_{29} \dot{\theta}_{3}^{B}=0
\end{aligned}
$$

where the coefficients $a_{i j}$ are functions of geometry and inertial parameters of RS.

During free motion, the applied joint actuator torques are internal. As a result, the linear momentum of RS in the plane and angular momentum normal to the plane remain constant. These three equations can be written as:

$$
\begin{aligned}
& m_{R S} \dot{x}_{R S}=K_{1} \\
& m_{R S} \dot{y}_{R S}=K_{2} \\
& a_{33} \dot{\theta}_{0}+a_{34} \dot{\theta}_{1}^{A}+a_{35} \dot{\theta}_{2}^{A}+a_{36} \dot{\theta}_{3}^{A}+a_{37} \dot{\theta}_{1}^{B}+a_{38} \dot{\theta}_{2}^{B} \\
& \quad+a_{39} \dot{\theta}_{3}^{B}=K_{3}
\end{aligned}
$$

where $m_{R S}$ is the mass of the robot system and $K_{1}$, $K_{2}, K_{3}$ are constant values of momentum components during free motion. These equations do not 
hold when the robot is acted on by external forces during propulsion, docking, and collision.

\subsubsection{Robot System with Locked Joints}

With the six joints locked, RS becomes a single rigid body. Hence, $x_{0, R S}, y_{0, R S}$ become dependent on $x_{R S}, y_{R S}$, and $\theta_{0}$. Hence, it is more convenient to describe the robot system by 3 independent variables: $x_{R S}, y_{R S}$, and $\theta_{0}$. In order to facilitate the developments of this paper, we define a vector $\mathbf{X}_{R S}=\left(x_{R S}, y_{R S}, \theta_{0}\right)^{T}$. Unless acted on by external forces or impacts, $\dot{\mathbf{X}}_{R S}$ remains constant.

\subsection{Table systems}

We define the vector $\mathbf{X}_{M S}=\left(x_{M S}, y_{M S}, \theta_{M S}\right)^{T}$ to describe the motion of the material system and $\mathbf{X}_{A S}=\left(x_{A S}, y_{A S}, \theta_{A S}\right)^{T}$ to describe the motion of the assembly table system. The rates $\dot{\mathbf{X}}_{M S}$ remain constant during motion unless MS is acted on by external forces or there is collision. Similarly, $\dot{\mathbf{x}}_{A S}$ remain constant during motion when the assembly table is not acted on by external forces.

\section{Models of Assembly Primi- tives}

In this paper, we will address the following assembly primitives: (i) Self motion of the robot system, (ii) Propulsion of the robot system, (iii) Docking of the robot system, (iv) Pickup of a workpiece by the robot system, and ( $v$ ) Release of a workpiece by the robot system. As mentioned earlier, the joints of the robot are locked during propulsion, docking, pickup, and release and are actively coordinated during self motion.

\subsection{Self Motion of the Robot System}

During self motion of the robot, with prescribed motion of the six joint angles, the time histories of base coordinates $x_{0, R S}$ and $y_{0, R S}$ are computed using Eqs. (2), (3). The position of the center of mass is governed by (4) and (5) and the orientation angle $\theta_{0}$ is computed using (6). The center of mass of RS drifts with a constant velocity during self motion.

\subsection{Propulsion of the Robot System}

The robot is propelled by 2 air thrusters placed at $T_{1}$ and $T_{2}$ on the base of the robot. The rates of RS during propulsion satisfy the following relation:

$$
M_{R S} \ddot{\mathbf{X}}_{R S}=J v\left(T_{1}\right)^{T} \mathbf{F}\left(T_{1}\right)+J v\left(T_{2}\right)^{T} \mathbf{F}\left(T_{2}\right)
$$

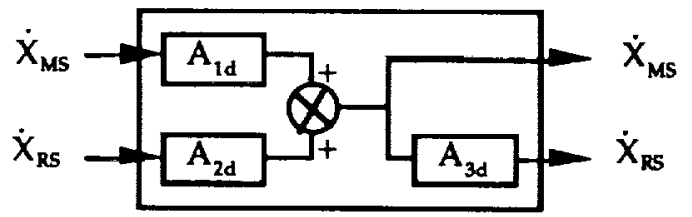

Figure 5: A block diagram of the rate relations for the dock primitive.

where $M_{R S}$ is the inertia matrix of the robot system with respect to $\mathbf{X}_{R S}, J v\left(T_{1}\right)$ and $J v\left(T_{2}\right)$ are respectively the velocity Jacobians for the thruster locations $T_{1}$ and $T_{2}$ with respect to $\dot{\mathbf{X}}_{R S} . \mathbf{F}\left(T_{1}\right)$ and $\mathbf{F}\left(T_{2}\right)$ are $(2 \times 1)$ thrust vectors described in $\mathcal{F}$.

Given $\mathbf{X}_{R S}, \dot{X}_{R S}$ at initial and final positions, time histories of the thruster forces $\mathbf{F}\left(T_{1}\right)$ and $\mathbf{F}\left(T_{2}\right)$ can be selected in a number of ways to satisfy the conditions at the two end points. A relatively simple way to achieve this is by selecting cubic trajectories for $\mathbf{X}_{R S}$ components that satisfy the end conditions. $\ddot{\mathbf{X}}_{R S}$ computed from these cubic trajectories can then be used to determine the thrust vectors as functions of time.

\subsection{Docking of the Robot System}

Assume that RS docks with MS such that after docking a point $P$ of RS acquires the same velocity as $P^{\prime}$ of MS and the two systems after docking acquire the same angular velocity. The analytical model of this primitive is based on collision theory between two rigid bodies [8]. The equations of impact for RS can be written as:

$$
M_{R S}\left(\left.\dot{\mathbf{X}}_{R S}\right|_{t+}-\left.\dot{\mathbf{X}}_{R S}\right|_{t-}\right)=-J_{P}^{T} \int_{t_{-}}^{t+} \mathbf{F}_{d} d t
$$

where $J_{P}$ is the Jacobian matrix of $\mathrm{P}$ on RS, and $\mathbf{F}_{d}$ is the collision vector $\left(F_{d x}, F_{d y}, M_{d z}\right)^{T}$ expressed in $\mathcal{F} . t+$ and $t$ - are respectively the time instances after and before collision. A similar equation for MS is:

$$
M_{M S}\left(\left.\dot{\mathbf{X}}_{M S}\right|_{t+}-\left.\dot{\mathbf{X}}_{M S}\right|_{t-}\right)=J_{P}^{\prime T} \int_{t-}^{t+} \mathbf{F}_{d} d t
$$

where $M_{M S}$ is the inertia matrix of MS for $\mathbf{X}_{M S}$ and $J_{P^{\prime}}$ is the Jacobian matrix of point $P^{\prime}$ on MS. After impact, $\dot{\mathbf{X}}_{R S}$ and $\dot{\mathbf{X}}_{M S}$ are related as follows:

$$
\left.J_{P} \dot{\mathbf{X}}_{R S}\right|_{t+}=J_{P},\left.\dot{\mathbf{X}}_{M S}\right|_{t+}
$$

On simultaneously solving these three equations, we obtain:

$$
\begin{array}{r}
\left.\dot{\mathbf{X}}_{M S}\right|_{t+}=\left[M_{M S}+J_{P^{\prime}}{ }^{T} J_{P}{ }^{-T} M_{R S} J_{P}{ }^{-1} J_{P^{\prime}}\right]^{-1} \\
{\left[\left.M_{M S} \dot{\mathbf{X}}_{M S}\right|_{t-}+\left.J_{P^{\prime}}{ }^{T} J_{P}{ }^{-T} M_{R S} \dot{\mathbf{X}}_{R S}\right|_{t}-(1)\right.}
\end{array}
$$




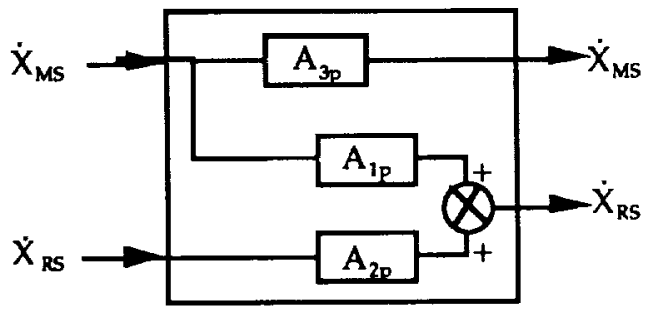

Figure 6: A block diagram of the rate relations for the 'pick' primitive.

and

$$
\left.\dot{\mathbf{X}}_{R S}\right|_{t+}=J_{P}^{-1} J_{P},\left.\dot{\mathbf{X}}_{M S}\right|_{t+}
$$

In order to concisely write the above two equations, where $A_{1 p}, A_{2 p}$ are defined as:

we define the following matrices:

$$
\begin{aligned}
A_{1 d} & =\left[M_{M S}+J_{P^{\prime}}{ }^{T} J_{P}{ }^{-T} M_{R S} J_{P^{-1}} J_{P^{\prime}}\right]^{-1} M_{M S} \\
A_{2 d} & =\left[M_{M S}+J_{P^{\prime}} T J_{P}{ }^{-T} M_{R S} J_{P^{-1} J_{P^{\prime}}}\right]^{-1} J_{P^{\prime}}{ }^{T} \\
J_{P} & { }^{-T} M_{R S} \\
A_{3 d} & =J_{P}{ }^{-1} J_{P^{\prime}}
\end{aligned}
$$

The rates of the two sytem can then be written as:

$$
\begin{aligned}
& \left.\dot{\mathbf{X}}_{M S}\right|_{t+}=\left.A_{1 d} \dot{\mathbf{X}}_{M S}\right|_{t-}+\left.A_{2 d} \dot{\mathbf{X}}_{R S}\right|_{t-} \\
& \left.\dot{\mathbf{X}}_{R S}\right|_{t+}=\left.A_{3 d} \dot{\mathbf{X}}_{M S}\right|_{t+}
\end{aligned}
$$

A block diagram of the docking primitive is shown in Fig. 5. It must be noted that $M_{R S}$ and $M_{M S}$ depend on the definitions of the two systems at $t-$, $J_{P^{\prime}}$ also depends on location of $P^{\prime}$ on MS, and $J_{P}$ depends on joint angles of RS.

\subsection{Object Pickup by the Robot Sys- tem}

Once RS has docked with MS and is ready to pickup $W_{i}$, this primitive relates the rates of RS and MS before and after pickup. It is assumed that during pickup the applied forces are normal to the plane of motion. The changes in the rates, therefore, occur due to redefinition of the two systems RS and MS. In the new definition, $W_{i}$ is added to RS and $W_{i}$ has been taken away from MS.

As a result of adding $W_{i}$ to $\mathrm{RS}$, it has a new position and velocity of the center of mass. The position of the new center of mass of RS is computed from the positions of $C_{R S *}$ and $C_{W i *}$.

$$
\begin{aligned}
& \left.x_{R S}\right|_{t+}=\frac{\left.\left.m_{R S}\right|_{t-} x_{R S}\right|_{t-}+\left.m_{W i} x_{W i *}\right|_{t-}}{\left.m_{R S}\right|_{t-}+m_{W i}} \\
& \left.y_{R S}\right|_{t+}=\frac{\left.\left.m_{R S}\right|_{t-} y_{R S}\right|_{t-}+\left.m_{W i} y_{W i *}\right|_{t-}}{\left.m_{R S}\right|_{t-}+m_{W i}}(15)
\end{aligned}
$$

The velocity of the new center of mass is computed from the velocities of $C_{R S *}$ and $C_{W i *}$.

$$
\begin{aligned}
& \left.\dot{x}_{R S}\right|_{t+}=\frac{\left.\left.m_{R S}\right|_{t-} \dot{x}_{R S}\right|_{t-}+\left.m_{W i} \dot{x}_{W i *}\right|_{t-}}{\left.m_{R S}\right|_{t-}+m_{W i}} \\
& \left.\dot{y}_{R S}\right|_{t+}=\frac{\left.\left.m_{R S}\right|_{t-} \dot{y}_{R S}\right|_{t-}+\left.m_{W i} \dot{y}_{W i *}\right|_{t-}}{\left.m_{R S}\right|_{t-}+m_{W i}}(16)
\end{aligned}
$$

The angular rate does not change as a result of pickup becuase the acting forces are normal to the plane of motion. Hence, $\left.\dot{\theta}_{R S}\right|_{t+}=\left.\dot{\theta}_{R S}\right|_{t-\text {. }}$ Using the velocity Jacobian of $C_{W i *}$, the rates before and after pickup can be related as:

$$
\left.\dot{\mathbf{X}}_{R S}\right|_{t+}=\left.A_{1 p} \dot{\mathbf{X}}_{R S}\right|_{t-}+\left.A_{2 p} \dot{\mathbf{X}}_{M S}\right|_{t-}
$$

$$
\begin{aligned}
& A_{1 p}=\left[\begin{array}{rrr}
\frac{m_{R S l_{t}}}{\left.m_{R S}\right|_{t-}+m_{W_{i}}} & 0 & 0 \\
0 & \frac{m_{\left.R S\right|_{t}}}{m_{\left.R S\right|_{t}+m_{W_{i}}}} & 0 \\
0 & 0 & 1
\end{array}\right]
\end{aligned}
$$

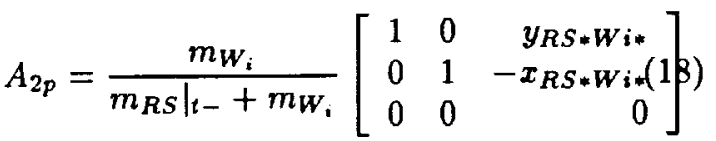

and $\left(x_{R S * W i_{*}}, y_{R S * W i_{*}}\right)$ are $\mathrm{X}$ and $\mathrm{Y}$ components of the vector from $C_{R S *}$ to $C_{W i *}$ expressed in $\mathcal{F}$.

As a result of losing $W_{i}$, MS has a new position and velocity of the center of mass. The new center of mass is computed from the positions of $C_{M S *}$ and $C_{W i *}$.

$$
\begin{aligned}
& \left.x_{M S}\right|_{t+}=\frac{\left.\left.m_{M S}\right|_{t-} x_{M S}\right|_{t-}-\left.m_{W i} x_{W i *}\right|_{t-}}{\left.m_{M S}\right|_{t-}-m_{W i}} \\
& \left.y_{M S}\right|_{t+}=\frac{\left.\left.m_{M S}\right|_{t-} y_{M S}\right|_{t-}+\left.m_{W i} y_{W i *}\right|_{t}}{\left.m_{M S}\right|_{t-}-m_{W i}}(19)
\end{aligned}
$$

Similarly, velocity of new $C_{M S_{*}}$ is computed from the velocities of the old $C_{M S *}$ and $W_{i}$.

$$
\begin{aligned}
& \left.\dot{x}_{M S}\right|_{t+}=\frac{\left.\left.m_{M S}\right|_{t-} \dot{x}_{M S}\right|_{t-}-\left.m_{W i} \dot{x}_{W i *}\right|_{t-}}{\left.m_{M S}\right|_{t-}-m_{W i}} \\
& \left.\dot{y}_{M S}\right|_{t+}=\frac{\left.\left.m_{M S}\right|_{t-} \dot{y}_{M S}\right|_{t-}-\left.m_{W i} \dot{y}_{W i *}\right|_{t}}{\left.m_{M S}\right|_{t-}-m_{W i}}(20)
\end{aligned}
$$

The two rate relations can be restructured in a matrix form:

$$
\left.\dot{\mathbf{X}}_{M S}\right|_{t+}=\left.A_{3 p} \dot{\mathbf{X}}_{M S}\right|_{t-}
$$

where $A_{3 p}$ is defined as:

$$
A_{3 p}=\left[\begin{array}{rrr}
k_{1}-k_{2} & 0 & -k_{2} y_{M S * W i *} \\
0 & k_{1}-k_{2} & k_{2} x_{M S * W i *} \\
0 & 0 & 0
\end{array}\right]
$$

where $k_{1}=\frac{m_{\left.M S\right|_{t-}}}{m_{\left.M S\right|_{t-}-m_{W_{1}}}}, k_{2}=\frac{m_{W_{i}}}{\left.m_{M S}\right|_{t-}-m_{W_{1}}}$, and $\left(x_{R M * W i *}, y_{R M * W i *}\right)$ are $\mathrm{X}$ and $\mathrm{Y}$ components 


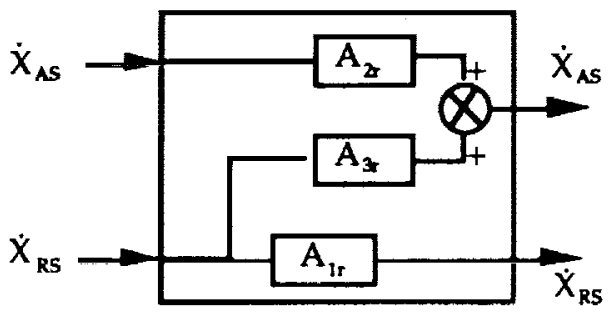

Figure 7: A block diagram of the rate relations for the 'release' primitive.

of the vector from $C_{M S *}$ to $C_{W i *}$ expressed in $\mathcal{F}$. A block diagram of this primitive is shown in Figure 6 . From this block diagram, we can notice that out of

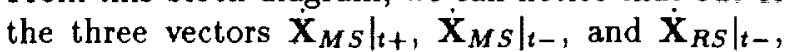
any two can be chosen independently. For example, if $\left.\dot{\mathbf{X}}_{M S}\right|_{t+}$ and $\left.\dot{\mathbf{X}}_{M S}\right|_{t-}$ are specified $\left.\dot{\mathbf{X}}_{R S}\right|_{t-}$ and $\left.\dot{\mathbf{X}}_{R S}\right|_{t+}$ can be uniquely determined.

\subsection{Release of an Object by the Robot System}

This primitive relates the rates of RS and AS once RS releases an object $W_{i}$ on AS. It is assumed that during release the applied forces are normal to the plane of motion. The changes in the rates, therefore, occur only due to redefinition of the systems.

As a result of removing $W_{i}$ from RS, it has a new position and velocity of the center of mass. The position of the new center of mass of RS is computed from the positions of $C_{R S *}$ and $C_{W i *}$.

$$
\begin{aligned}
& \left.x_{R S}\right|_{t+}=\frac{\left.\left.m_{R S}\right|_{t-} x_{R S}\right|_{t-}-\left.m_{W i} x_{W i}\right|_{t-}}{\left.m_{R S}\right|_{t-}-m_{W i}} \\
& \left.y_{R S}\right|_{t+}=\frac{\left.\left.m_{R S}\right|_{t-} y_{R S}\right|_{t-}-\left.m_{W i} y_{W i}\right|_{t-}}{\left.m_{R S}\right|_{t-}-m_{W i}}
\end{aligned}
$$

Similarly, the velocity of the new center of mass is:

$$
\begin{aligned}
& \left.\dot{x}_{R S}\right|_{t+}=\frac{\left.\left.m_{R S}\right|_{t-} \dot{x}_{R S}\right|_{t-}-\left.m_{W i} \dot{x}_{W i}\right|_{t-}}{\left.m_{R S}\right|_{t-}-m_{W i}} \\
& \left.\dot{y}_{R S}\right|_{t+}=\frac{\left.\left.m_{R S}\right|_{t-} \dot{y}_{R S}\right|_{t-}-\left.m_{W i} \dot{y}_{W i}\right|_{t-}}{\left.m_{R S}\right|_{t-}-m_{W i}}
\end{aligned}
$$

The two rate relations can be restructured in a matrix form:

$$
\left.\dot{\mathbf{X}}_{R S}\right|_{t+}=\left.A_{1, r} \dot{\mathbf{X}}_{R S}\right|_{t-}
$$

where $A_{1 r}$ is defined as:

$$
A_{3 p}=\left[\begin{array}{rrr}
k_{3}-k_{4} & 0 & -k_{4} y_{R S * W i *} \\
0 & k_{3}-k_{4} & k_{4} x_{R S * W i *} \\
0 & 0 & 0
\end{array}\right]
$$

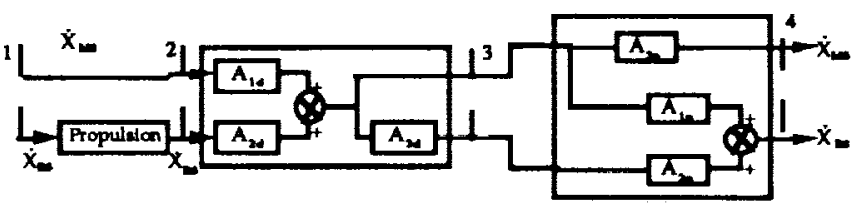

Figure 8: A block diagram of the 'propel/dock/pick' primitive.

where $k_{3}=\frac{m_{\left.R S\right|_{t}}}{m_{R S t_{-}-m_{W_{i}}}}, k_{4}=\frac{m_{W_{i}}}{m_{\left.R S\right|_{t-}-m_{W_{i}}}}$, and $\left(x_{R S * W i *}, y_{R S * W W_{*}}\right)$ are $\mathrm{X}$ and $\mathrm{Y}$ components of the vector from $C_{R S *}$ to $C_{W i *}$ expressed in $\mathcal{F}$.

The velocity of the new center of mass of AS is computed from the velocities of $C_{A S_{*}}$ and $C_{W_{i *}}$.

$$
\begin{aligned}
& \left.x_{A S}\right|_{t+}=\frac{\left.\left.m_{A S}\right|_{t-} x_{A S}\right|_{t-}+\left.m_{W i} x_{W i}\right|_{t-}}{\left.m_{A S}\right|_{t-}+m_{W i}} \\
& \left.y_{A S}\right|_{t+}=\frac{\left.\left.m_{A S}\right|_{t-} y_{M S}\right|_{t-}+\left.m_{W i} y_{W i}\right|_{t-}}{\left.m_{A S}\right|_{t-}+m_{W i}}
\end{aligned}
$$

Similarly, the new velocity of the center of mass is:

$$
\begin{aligned}
& \left.\dot{x}_{A S}\right|_{t+}=\frac{\left.\left.m_{A S}\right|_{t-} \dot{x}_{A S}\right|_{t-}+\left.m_{W_{i}} \dot{x}_{W i}\right|_{t-}}{\left.m_{A S}\right|_{t-}+m_{W i}} \\
& \left.\dot{y}_{A S}\right|_{t+}=\frac{\left.\left.M_{A S}\right|_{t-} \dot{y}_{A S}\right|_{t-}+\left.m_{W i} \dot{y}_{W i}\right|_{t-}}{\left.m_{A S}\right|_{t-}+m_{W i}}
\end{aligned}
$$

The angular rate does not change as a result of adding $W_{i}$, hence, $\left.\dot{\theta}_{A S}\right|_{t+}=\left.\dot{\theta}_{A S}\right|_{t-\text {. Using the }}$ velocity Jacobian of $C_{W i *}$, the rates before and after pickup can be related as:

$$
\left.\dot{\mathbf{X}}_{A S}\right|_{t+}=\left.A_{2 r} \dot{\mathbf{X}}_{A S}\right|_{t-}+\left.A_{3 r} \dot{\mathbf{X}}_{R S}\right|_{t-}
$$

where $A_{2 r}, A_{3 r}$ are defined as:

$$
\begin{aligned}
A_{2 r} & =\left[\begin{array}{rrrr}
\frac{m_{\left.A S\right|_{t-}}}{m_{\left.A S\right|_{t-}+m_{W_{i}}}} & & 0 & 0 \\
0 & \frac{m_{\left.A S\right|_{t-}}}{m_{\left.A S\right|_{t-}+m_{W_{i}}}} & 0 \\
0 & 0 & 1
\end{array}\right] \\
A_{3 r} & \left.=\frac{m_{W_{i}}}{\left.m_{A S}\right|_{t-}+m_{W}}\left[\begin{array}{rrrr}
1 & 0 & y_{A S * W i *} \\
0 & 1 & -x_{A S * W i *} \\
0 & 0 & 0
\end{array}\right]\right)
\end{aligned}
$$

and $\left(x_{A S * W i_{*}}, y_{A S * W i *}\right)$ are $\mathrm{X}$ and $\mathrm{Y}$ components of the vector from $C_{A S_{*}}$ to $C_{W i *}$ expressed in $\mathcal{F}$. A block diagram of this primitive is shown in Figure 7.

\section{Modeling of Assembly}

\subsection{A Simple Assembly}

Consider a simple assembly task that requires the robot to pick $W_{1}$ and $W_{2}$ from MS, assemble these in the form of an ' $L$ ' shape, and place this composite body on AS. A possible sequence of primitives 


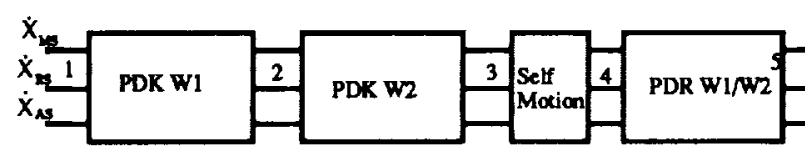

Figure 9: A block diagram of the simple assembly described in Section 4.

to complete this assembly task is: (i) RS propels to $W_{1}$, (ii) RS docks with MS to grip $W_{1}$ by $\operatorname{arm} \mathrm{A}$, (iii) RS picks $W_{1}$ from MS, (iv) RS propels to $W_{2}$, (v) RS docks with MS to grip $W_{2}$ by arm $\mathrm{B}$, (vi) RS picks $W_{2}$ from MS, (vii) RS executes self motion to assemble $W_{1}$ and $W_{2}$, (viii) RS propels to AS, (ix) RS docks with AS to release $W_{1} / W_{2},(x)$ RS releases $W_{1} / W_{2}$ on AS. In this small assembly task, we saw the sequence of primitives propel, dock, and pickup (PDK) repeated twice and the sequence propel, dock, and release (PDR) once. These two sequences of primitives appear quite commonly during assembly and require further study to determine their characteristics.

\subsection{Propulsion/dock/pickup (PDK) Sequence}

Fig. 8 shows a block diagram of this sequence of primitives. It can be infered from this block diagram that if $\dot{\mathrm{X}}_{M S}$ at nodes 1 and 4 are specified, $\dot{\mathbf{X}}_{M S}$ at nodes 2,3 and $\dot{\mathbf{X}}_{R S}$ at nodes 2, 3, 4 are uniquely determined. Also, with the propulsion primitive, for any given $\mathbf{X}_{R S}$ and $\dot{\mathbf{X}}_{R S}$ at node 1, a desired $\mathbf{X}_{R S}, \dot{\mathbf{X}}_{R S}$ at node 2 can be reached by suitably selecting a time history of the thruster forces. From these two observations, one can form a broader conclusion that it is possible to achieve any desired $\dot{\mathbf{X}}_{M S}$ at the end of a PDK sequence for arbitrary $\dot{\mathbf{X}}_{M S}$ and $\dot{\mathbf{X}}_{R S}$ at the beginning of the sequence. A similar conclusion can be made for propulsion/dock/release (PDR) sequence. These two conclusions play important roles in developing strategies for assembly.

\section{Description of FLOAT}

A general purpose program FLOAT was developed to study and test a variety of assembly strategies in a free-floating planar work environment. The inputs to this program consist of (i) the inertial description of the units, (ii) the geometric description of the units, (iii) the assembly sequence in the form

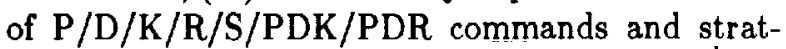
egy of assembly in terms of desired values of $\dot{\mathrm{X}}_{R S}$, $\dot{\mathbf{X}}_{M S}, \dot{\mathbf{X}}_{A S}$ at different points of the assembly sequence. The program creates the current RS, MS,

\section{Conclusions}

In this paper, we presented a method for analytical modeling of assembly using a free-floating planar robot in a free-floating planar work environment. The model of the assembly was obtained by combining analytical models of five primitives: (i) self motion of the robot, (ii) propulsion of the robot, (iii) docking of the robot, (iv) pickup by the robot, and (v) release by the robot. It was concluded that assemblies typically consist of a number of propulsion, dock, pickup/release sequences interluded by self motion. On examining a PDK sequence, it was observed that starting out from arbitrary velocities of the robot system and material system, it was possible to achieve any desired material system velocities by suitably controlling the thruster forces of the robot system during propulsion. A similar conclusion could be arrived at for a PDR sequence. These observations provide guidelines to select proper velocities of RS, MS, AS at intermediate steps during assembly. A general purpose program was developed to study and test assembly strategies for a variety of assemblies. Even though this paper deals specifically for planar free-floating robots, the concepts can be extended to free-floating spatial robots working in zero gravity environment. 


\section{Acknowledgments}

The support of the Dynamic Systems and Control Division of the National Science Foundation under the Research Initiation Program, 1804 Fund Awards, Baker Fund Awards from Ohio University are gratefully acknowledged.

\section{Appendix}

\subsection{Jacobian for Points on a Rigid Body}

Consider a rigid body $B$ undergoing planar motion. The position of a point $B *$ on this body is described in an inertial frame $\mathcal{F}$ by the coordinates $x_{B *}$ and $y_{B *}$. The orientation of a line $B * B_{1}$ is described by the angle $\theta_{\boldsymbol{B}}$. The velocity Jacobian for point $B_{1}$ with respect to $\dot{x}_{B^{*}}, \dot{y}_{B^{*}}, \dot{\theta}_{B}$ in $\mathcal{F}$ is given as:

$$
\left[\begin{array}{r}
V_{B 1 x} \\
V_{B 1 y} \\
\dot{\theta}_{B}
\end{array}\right]=\left[\begin{array}{rrr}
1 & 0 & y_{B * B 1} \\
0 & 1 & -x_{B * B 1} \\
0 & 0 & 1
\end{array}\right]\left[\begin{array}{r}
\dot{x}_{B *} \\
\dot{y}_{B *} \\
\dot{\theta}_{B}
\end{array}\right]
$$

where $\left(x_{B * B 1} a n d y_{B * B 1}\right)^{T}$ are $\mathrm{X}$ and $\mathrm{Y}$ components of the vector $\mathrm{r}_{B * B 1}$ expressed in $\mathcal{F}$. The $(3 \times 3)$ matrix is the Jacobian map for point $B_{1}$ labeled as $J_{B_{1}}$. The upper $(2 \times 3)$ block is the velocity Jacobian $J v(B 1)$.

\subsection{Inertia Matrix for a Rigid Body}

In Section 8.1, if $B *$ is the center of mass of $\mathrm{B}$, the inertia matrix of body $B$ relative to the coordinates $\left(x_{B *}, y_{B *}, \theta_{B}\right)^{T}$ is given as:

$$
M_{B}=\left[\begin{array}{rrr}
m_{B} & 0 & 0 \\
0 & m_{B} & 0 \\
0 & 0 & I_{B}
\end{array}\right]
$$

where $m_{B}$ is the mass of the body $\mathrm{B}$ and $I_{B}$ is the centroidal moment of inertia about an axis normal to the plane of motion.

\section{References}

[1]
Agrawal, S.K. and Garimella, R.,"Workspace Boundaries of Free-floating Open and ClosedChain Planar Manipulators", to appear in Journal of Mechanical Design, Transactions of the ASME, 1993.

Agrawal, S.K. and Desmier, G., "Kinematics of Assembly Primitives for Free-floating Robots", In Proceedings, IEEE International Conference on Intelligent Robot Systems, Yokohama, Japan, 1993.

Agrawal, S.K. and Garimella, R., "Pathplanning of a Free-floating Closed-Chain Planar Manipulator Using Inverse Kinematics", under review, Journal of Mechanical Design, Transactions of the ASME, 1993.

Agrawal, S.K. and Shirumalla, S., ${ }^{\text {Motion planning of }}$ a Dual-arm Free-floating Planar Manipulator with Inertially Fixed Basen, to appear in Mechanism and Machine Theory, 1993.

Agrawal, S.K. and Krajnak, J.A., "Design of a Quadrilateral Pneumatic Thruster for a Free-floating Robot", under review IEEE Transactions on Robotics and Automation, 1993.

Agrawal, S.K., Rambhaskar, J., and Annapragada, M., "Design of a New Contactless Sensor for Robotic Applications", under review IEEE Transactions on Robotics and Automation, 1993.

Alexander, H.L. and Cannon, R.H., "An Extended Operational Space Control Algorithm for Satellite Manipulators", The Journal of Astronautical Sciences, vol. 38, no. 4, 1990, pp. 473-486.

Kane, T.R. and Levinson, D.A., Dynamics: Theory and Applications, McGrawHill Book Company, New York, 1985.

Nakamura, Y. and Mukherjee, R., "Nonholonomic Path Planning of Space Robots Via Biirectional Approach", IEEE Transactions on Robotics and Automation, vol. 7 , no. 4, 1991, pp. 500514.

Papadopoulos, E. and Dubowsky, S., "On the Dynamic Singularities in the Control of Space Manipulators", ASME Winter Annual Meeting, 1989, pp. 45-51.

[11] Schneider, S.A. and Cannon, R.H., "Object Impedance Control for Cooperative Manipulation: Theory and Experimental Results", IEEE Transactions on Robotics and Automation, vol. 8, June 1992, pp. 383-394. 
[12] Vafa, Z., "Space Manipulator with No Satellite Attitude Disturbance", IEEE International Conference on Robotics and Automation, 1990, pp. 1770-1775.

[13] Yoshida, K., Kurazume, R., and Umetani, Y., "Dual Arm Coordination in Space Free-flying Robot", In proceedings, IEEE International Conference on Robotics and Automation, vol.3, April 1991, pp. 2516-2521. 